\title{
PLANTAS VASCULARES DE MACARONESIA, EXCEPTO CANARIAS, EN EL HERBARIO TFC
}

\author{
Cristina González-Montelongo*, María Catalina León Arencibia*, \\ Jorge Alfredo Reyes-Betancort** \& Juan Ramón Acebes Ginovés*
}

\section{RESUMEN}

El Herbario TFC es el Herbario Institucional de la Universidad de La Laguna (ULL), Tenerife, Islas Canarias y alberga cinco colecciones principales: algas, hongos, líquenes, briófitos y plantas vasculares. La mayor de ellas es la de plantas vasculares con 53000 pliegos, 890 de ellos recolectados en los archipiélagos macaronésicos de Azores (45 pliegos), Madeira (401), Salvajes (44) y Cabo Verde (400), correspondientes a 91 familias (dominando Fabaceae, Asteraceae, Lamiaceae, Poaceae y Scrophulariaceae), 279 géneros (dominando Teline, Lavandula, Lotus, Micromeria y Genista), 396 especies, 19 subespecies, 26 variedades y 1 forma. Están representadas más del $50 \%$ de las familias botánicas presentes en los archipiélagos de Salvajes y Cabo Verde. El Herbario TFC presenta al menos un pliego de más de la mitad de endemismos de Cabo Verde y más del 40\% de los endemismos de Salvajes. PALABRAS ClAVE: herbario TFC, colección de historia natural, macaronesia, plantas vasculares.

\section{MACARONESIAN (EXCEPT CANARIES) VASCULAR PLANTS \\ IN THE TFC HERBARIUM}

Abstract

TFC Herbarium is the Institutional Herbarium of the University of La Laguna (ULL), Tenerife, Canary Islands. TFC herbarium houses five main collections: algae, fungi, lichens, bryophytes and vascular plants. The largest is vascular plants with 53000 sheets, 890 of them collected in Macaronesian archipelagos of Azores (45 sheets), Madeira (401), Selvagens (44) and Cabo Verde (400), of 91 families (principally Fabaceae, Asteraceae, Lamiaceae, Poaceae and Scrophulariaceae), 279 genera (principally Teline, Lavandula, Lotus, Micromeria and Genista), 396 species, 19 subspecies, 26 varieties and 1 form. More than $50 \%$ of the botanic families of Selvagens and Cabo Verde are present. TFC Herbarium house at least one sheet of more than half of Cabo Verde endemisms and more than $40 \%$ Selvagens endemisms.

KEYwORDs: TFC herbarium, natural history collection, macaronesia, vascular plants. 


\section{INTRODUCCIÓN}

Los herbarios son un recurso de vital importancia para los estudios taxonómicos de vegetales y hongos, siendo una fuente de información de biodiversidad, del presente y pasado de la flora de una determinada región. Actualmente muchos trabajos de evolución, genética, especies invasoras, biología de la conservación, cambio climático, entre otros, se apoyan tanto en el análisis de los especímenes depositados en herbarios como en los datos asociados a ellos (Grass et al. 2014, Davis et al. 2015, Yoshida et al. 2015, Gallinat et al. 2018). Estos estudios en auge han conllevado un cambio cualitativo y cuantitativo en el uso tradicional de los herbarios, indispensables cada vez más para otras disciplinas de la ciencia relacionadas con la Botánica, y que han puesto en valor al herbario como herramienta de investigación (Heberling y Isaac 2017). Desafortunadamente, los muestreos destructivos necesarios para algunos de los estudios anteriormente citados entran en conflicto con la conservación del material de herbario. Mientras el personal de los herbarios intenta salvaguardar la integridad de sus colecciones (Rabeler et al. 2019), los investigadores desarrollan y aplican nuevas técnicas que permiten desarrollar sus trabajos minimizando el daño producido al material, permitiendo un uso más eficiente de estas colecciones de historia natural (Shepherd 2017). En el caso del Herbario TFC, la normativa de muestreos destructivos es la aceptada por la Asociación de Herbarios Íbero-Macaronésicos (AHIM 2012).

En las últimas décadas han sido muchos los herbarios que se han sumado a la tarea de informatizar los datos de sus especímenes convencidos de la utilidad de los mismos para la comunidad científica. Tanto es así que se espera que en la próxima década los datos de los especímenes de herbario adquieran una importancia aún mayor tanto para la gestión como para la investigación (Baena 2005, Smith y Blagoderov 2012, Lavoie 2013, Holmes et al. 2016).

El Herbario TFC, fundado en 1969 (Thiers actualizado continuamente), es el Herbario Institucional de la Universidad de La Laguna (ULL), incluido en los Servicios Generales de Apoyo a la Investigación (SEGAI) y consta de 5 colecciones principales: algas, hongos, líquenes, briófitos y plantas vasculares. La colección de mayor tamańo es la de plantas vasculares, con unos 53000 pliegos. Aunque principalmente está dedicada a la flora canaria, presenta varias subcolecciones separadas por áreas geográficas: Europa, norte de África, América y Macaronesia, incluyendo esta última únicamente el material de los archipiélagos de Azores, Madeira, Salvajes y Cabo Verde. La tarea de informatización de la colección de plantas vasculares de este herbario se encuentra en una fase inicial (ver Trujillo-Trujillo et al. 2014).

* Departamento de Botánica, Ecología y Fisiología Vegetal, Facultad de Farmacia, Universidad de La Laguna (ULL), Islas Canarias, Spain. Corresponding autor: cgonzalm@ull.edu.es.

** Jardín de Aclimatación de La Orotava, Instituto Canario de Investigaciones Agrarias (ICIA), Islas Canarias, Spain. 
Siendo conscientes de la importancia de dar a conocer el patrimonio biológico de los archipiélagos macaronésicos depositado en el Herbario TFC, se ha llevado a cabo la informatización de los datos asociados a estos especímenes y su análisis. El objetivo principal de nuestra comunicación no es solo dar a conocer el material de esta colección depositado en el Herbario TFC sino incentivar su uso y consulta por parte de la comunidad científica.

\section{MATERIAL Y MÉTODOS}

Se han informatizado los datos correspondientes a cada pliego de la colección de Macaronesia, haciendo uso del Gestor de Colecciones Elysia 1.0 de GBIF. ES (Nodo español de "Global Biodiversity Information Facility»), con la intención de publicarlos en el Portal Nacional de Datos de Biodiversidad. Posteriormente, se analizaron los datos referentes a las localidades, hábitats, fecha de recolección, recolector, identificador y otras observaciones (herbario original de procedencia del material en el caso de donaciones, campañas de recolección, etc.), con la finalidad de estudiar la cobertura taxonómica, temporal y espacial siguiendo la metodología de García-Sánchez y Cabezudo (2013), así como la calidad de los datos y la importancia de esta colección de plantas vasculares, empleando los parámetros considerados como descriptores de la importancia de colecciones de herbarios con los que contábamos (Rich 1998). Finalmente, se han comparado los datos obtenidos a través del estudio del material incluido en el herbario con los listados oficiales de biodiversidad de los distintos archipiélagos macaronésicos (Sánchez-Pinto et al. 2005; Silva et al. 2005; Jardim y Menezes de Sequeira 2008), de los cuales se ha extraído información referente a la presencia insular y grado de endemicidad de las especies y subespecies con al menos un espécimen depositado en el Herbario TFC.

En el marco de este trabajo no se actualizó la nomenclatura utilizada por los especialistas que han identificado el material. Las actualizaciones nomenclaturales automáticas no son una práctica habitual en el trabajo rutinario del Herbario TFC. Estas se realizan tras la revisión y corrección de la identidad del material por parte de especialistas. Es por esto por lo que se ha mantenido en todo momento los datos que aparecen en las etiquetas originales, o en todo caso los de las etiquetas de revisión.

\section{RESULTADOS}

El Herbario TFC tiene 890 pliegos de plantas vasculares de los archipiélagos de Azores, Madeira, Salvajes y Cabo Verde. 


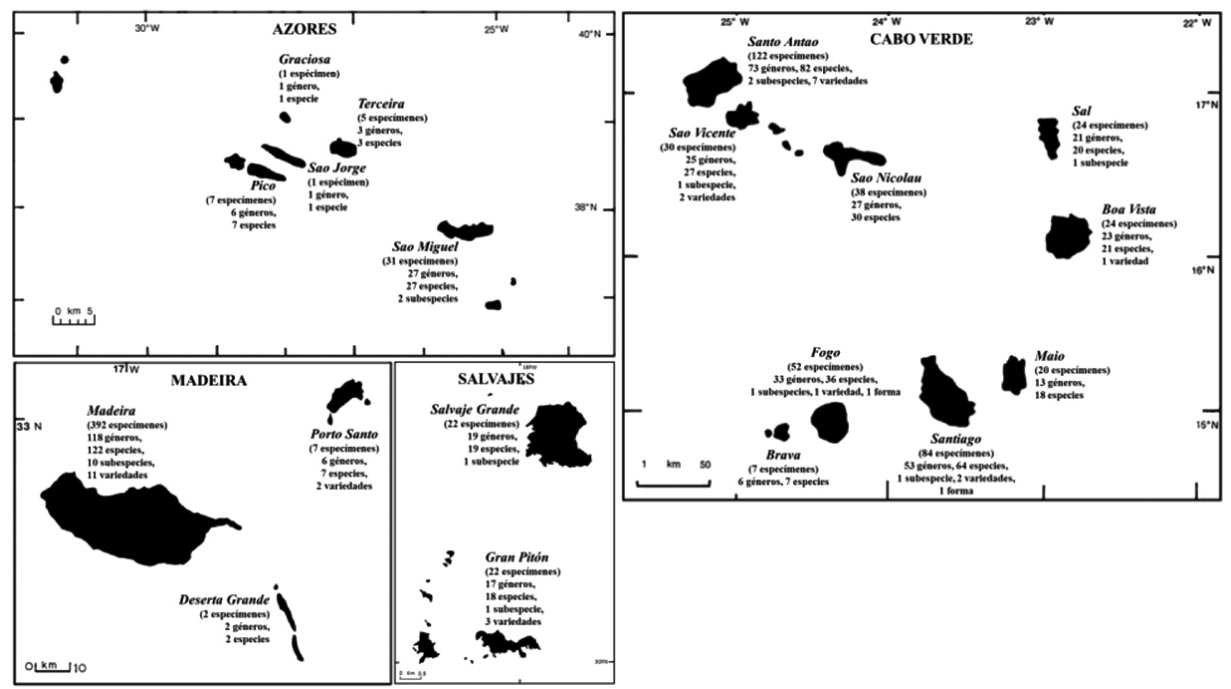

Figura 1. Cobertura espacial de la colección de plantas vasculares de Macaronesia (excepto Canarias) del Herbario TFC. Archipiélagos de Azores, Madeira, Salvajes y Cabo Verde con número de especímenes y taxones por isla presentes en el Herbario TFC.

Los archipiélagos con mayor número de pliegos en el Herbario TFC son Madeira (401 pliegos) y Cabo Verde (400), seguidos de Azores (45) y Salvajes (44). Hemos encontrado material procedente de 5 islas de Azores (Graciosa, Pico, Terceira, São Jorge y São Miguel), de 3 islas de Madeira (Madeira, Porto Santo y Deserta Grande), de 2 islas de Salvajes (Salvaje Grande y Salvaje Pequeña o Gran Pitón), y de 9 islas de Cabo Verde (Santo Antão, São Vicente, São Nicolau, Sal, Boa Vista, Maio, Santiago, Fogo y Brava) (figura 1).

\subsection{Cobertura taXonómica}

El material estudiado corresponde a 91 familias, 279 géneros, 396 especies, 19 subespecies, 26 variedades y 1 forma. Las familias con mayor número de especímenes son Fabaceae (170 pliegos), Asteraceae (114), Lamiaceae (106), Poaceae (33) y Scrophulariaceae (31). Están representadas más del 50\% de las familias botánicas presentes en los archipiélagos de Salvajes $(60 \%)$ y Cabo Verde $(50,5 \%)$, así como el 40,6\% de las presentes en Madeira y un 19,8\% de las familias presentes en Azores. Los géneros con mayor número de pliegos son Teline (50 pliegos), Lavandula (28), Lotus (28), Micromeria (27) y Genista (18), siendo los de mayor 
A

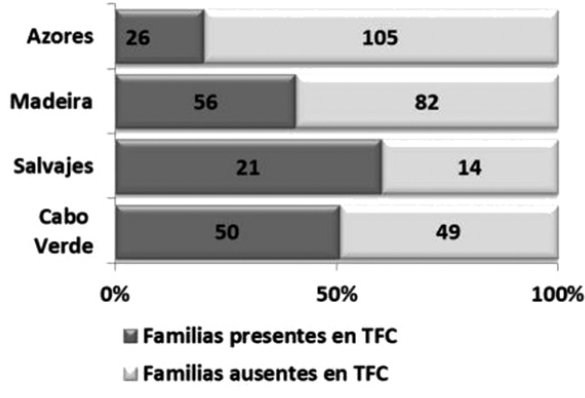

C

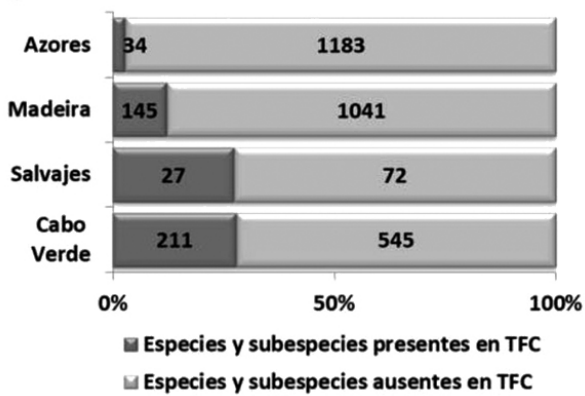

B

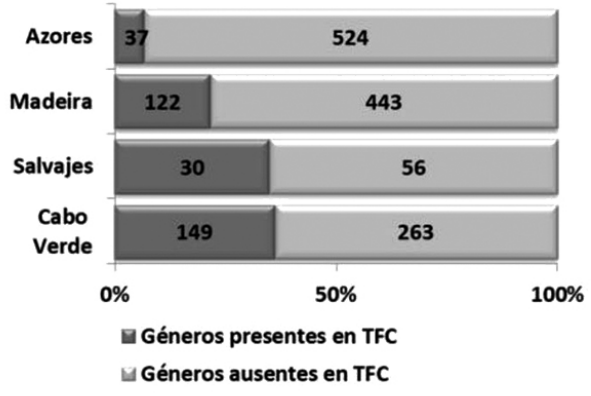

D

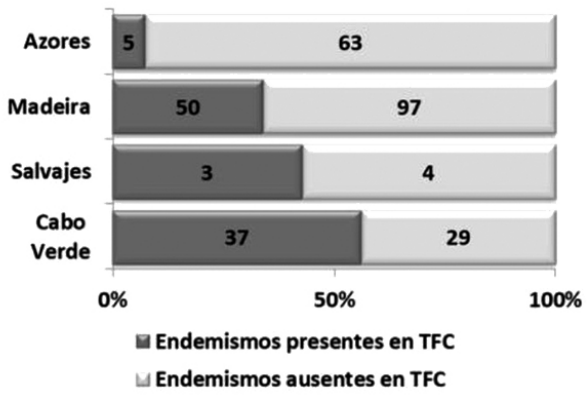

Figura 2. Cobertura taxonómica de la colección de plantas vasculares de Macaronesia (excepto Canarias) del Herbario TFC. Análisis del porcentaje (valores absolutos especificados en las barras) de familias (A), géneros (B), especies y subespecies (C) y endemismos (D) de cada archipiélago macaronésico presentes en el Herbario TFC.

diversidad Cyperus (con 9 taxones representados), Lotus (8), Micromeria (7), Lavandula (7) y Polygonum (6). Destaca igualmente la representación de géneros de los archipiélagos de Cabo Verde (36,2\%) y Salvajes (34,9\%) en el Herbario, mientras que los porcentajes de representación de géneros son igualmente menores para los archipiélagos de Madeira (21,6\%) y Azores (6,6\%). Como era de esperar, la representación específica y subespecífica de cada archipiélago disminuye en porcentajes respecto al de géneros y familias, pero siguen el mismo patrón, destacando como mejor representadas en el Herbario TFC las especies y subespecies de Cabo Verde $(27,9 \%)$ y Salvajes (27,3\%), seguidas por las de Madeira (12,2\%) y Azores (2,8\%). Por último, y algo más ligado a la conservación de la biodiversidad, es de destacar que el 56,1\% de los endemismos de Cabo Verde se encuentran representados con al menos un espécimen en este Herbario. El porcentaje de endemicidad disminuye hasta un 42,9\% para el archipiélago de Salvajes, seguido de un 34\% para Madeira y un $7,4 \%$ para Azores (figura 2). 
A

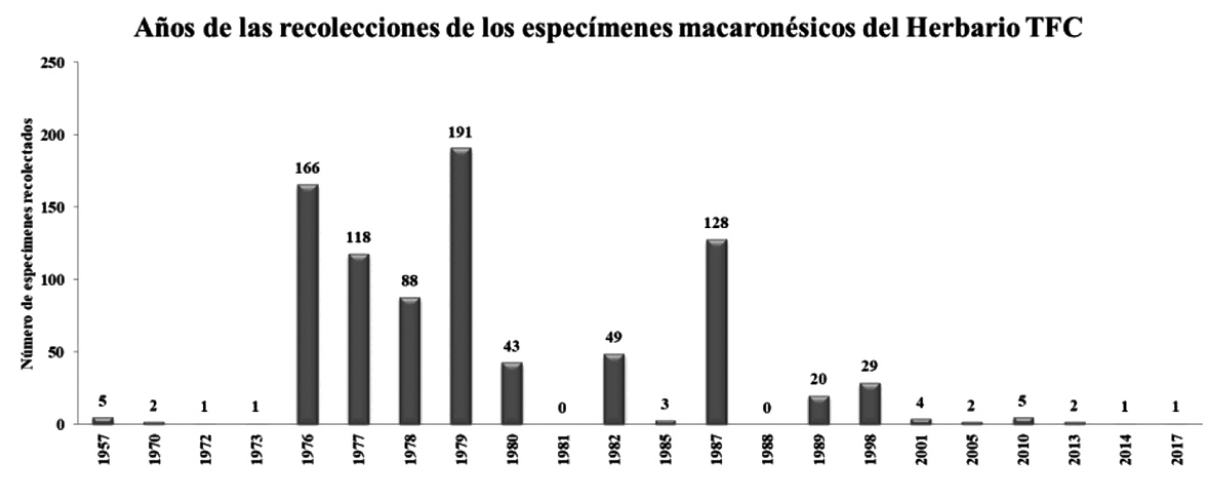

B

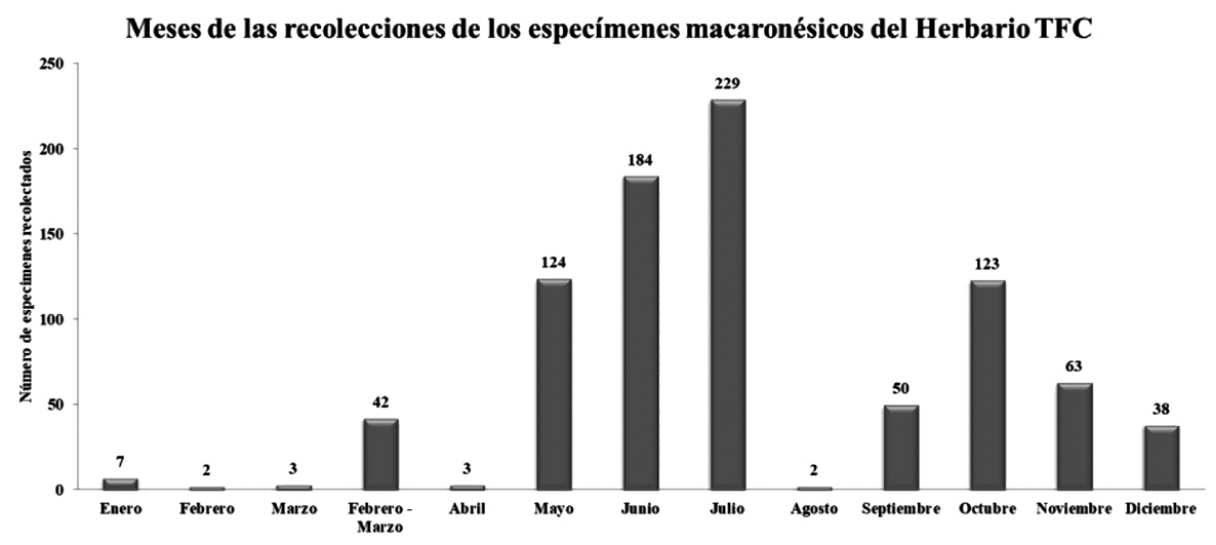

Figura 3. Cobertura temporal de la colección de plantas vasculares de Macaronesia, excepto Canarias, del Herbario TFC. Registro de los ańos (A) y los meses (B) en los que se han realizado las recolecciones del material. La presencia de un mes extra correspondiente a febrero-marzo se debe a las recolecciones realizadas en la expedición «Agamenón 76» (Salvajes), cuyo material no presenta la fecha exacta de la recolección.

\subsection{Cobertura temporal}

Las recolecciones más antiguas corresponden a las realizadas por João Vicente Cordeiro Malato-Beliz (1920-1993) en 1957, en Madeira. La mayor producción en cuanto al número de ejemplares recolectados e incorporados finalmente en el TFC, corresponden a los años 70 y 80 del siglo pasado (figura 3a), desarrollándose la mayoría de las recolecciones en los meses de mayo a julio y octubre (figura $3 \mathrm{~b}$ ). 


\subsection{Calidad de los datos E importancia de la colección}

La política del Herbario TFC es incorporar material cuando esté determinado, como mínimo, a nivel genérico. Tan solo 137 especímenes se encuentran identificados únicamente a nivel genérico, de los cuales solo 6 especímenes no tienen el género confirmado. El 29,9\% del material de esta colección se encuentra sin confirmar su identidad, bien sea a nivel genérico, específico o subespecífico. Prácticamente todo material identificado a nivel específico o infraespecífico presenta la autoría del taxón (faltando tan solo en 58 registros). Lo mismo ocurre con los datos referentes al lugar de recolección, fecha de recolección y recolector. Por el contrario, la mayoría del material no presenta especificada la autoría de la determinación ni la fecha en la que fue identificado, faltando estos datos en 718 y 853 pliegos, respectivamente. En algunos casos, se puede asignar la identificación al recolector, pero cuando hay varios recolectores, la tarea de identificación no suele ser fácilmente asignable a una única persona.

La colección ha sido objeto de consulta por parte de especialistas y como consecuencia de ello, la identidad de 23 especímenes ha sido corregida. Tan solo una pequeña parte del material presenta al menos un duplicado establecido. Además, el Herbario TFC es el depositario del Holotypus de Micromeria varia Bentham subsp. thymoides (Solander ex Lowe) P. Pérez var. cacuminicolae P. Pérez. Otros dos pliegos son materiales testigos de otro tipo de estudio: el TFC 11304 (Lavandula pinnata L.f.) es material testigo de estudio palinológico (muestra P-TFC 695 de la Palinoteca del Herbario TFC) y el TFC 52657 (Artemisia gorgonum Webb) es testigo de estudio de hongos endófitos. Más de la mitad del material (472 especímenes) procede de otros herbarios, tanto institucionales como privados (figura 4).

\section{DISCUSIÓN}

Hasta ahora, la consulta de los datos correspondientes al material de plantas vasculares recolectado en los archipiélagos macaronésicos requería del estudio del fichero, en soporte físico, o de los pliegos. Gracias a la tarea de informatización llevada a cabo, tanto la consulta como el análisis de estos datos serán más ágiles.

Se verifica el sesgo de recolección espacial, hacia los archipiélagos de Madeira y Cabo Verde, siendo el de Azores el peor representado en el Herbario TFC, así como un sesgo de recolección taxonómica para los géneros Teline y Genista en cuanto a número de recolecciones, Cyperus y Polygonum en cuanto a número de taxones representados y Lavandula, Lotus y Micromeria tanto por el número de recolecciones como por el número de taxones representados, recolectado en gran parte en el marco de las tesis doctorales de Pérez de Paz (1978), Del Arco Aguilar (1981) y León Arencibia (1982). La donación de las recolecciones realizadas por Wolfram Lobin en Cabo Verde, de 1978 a 1982, con motivo de su tesis doctoral (Lobin 1982), explica el número de pliegos de Cabo Verde presentes (261 pliegos), lo que explica además los elevados porcentajes de familias, géneros, especies y subespecies caboverdianos, con elevado porcentaje de endemicidad, representados en TFC (Lobin 1982 y Bro- 


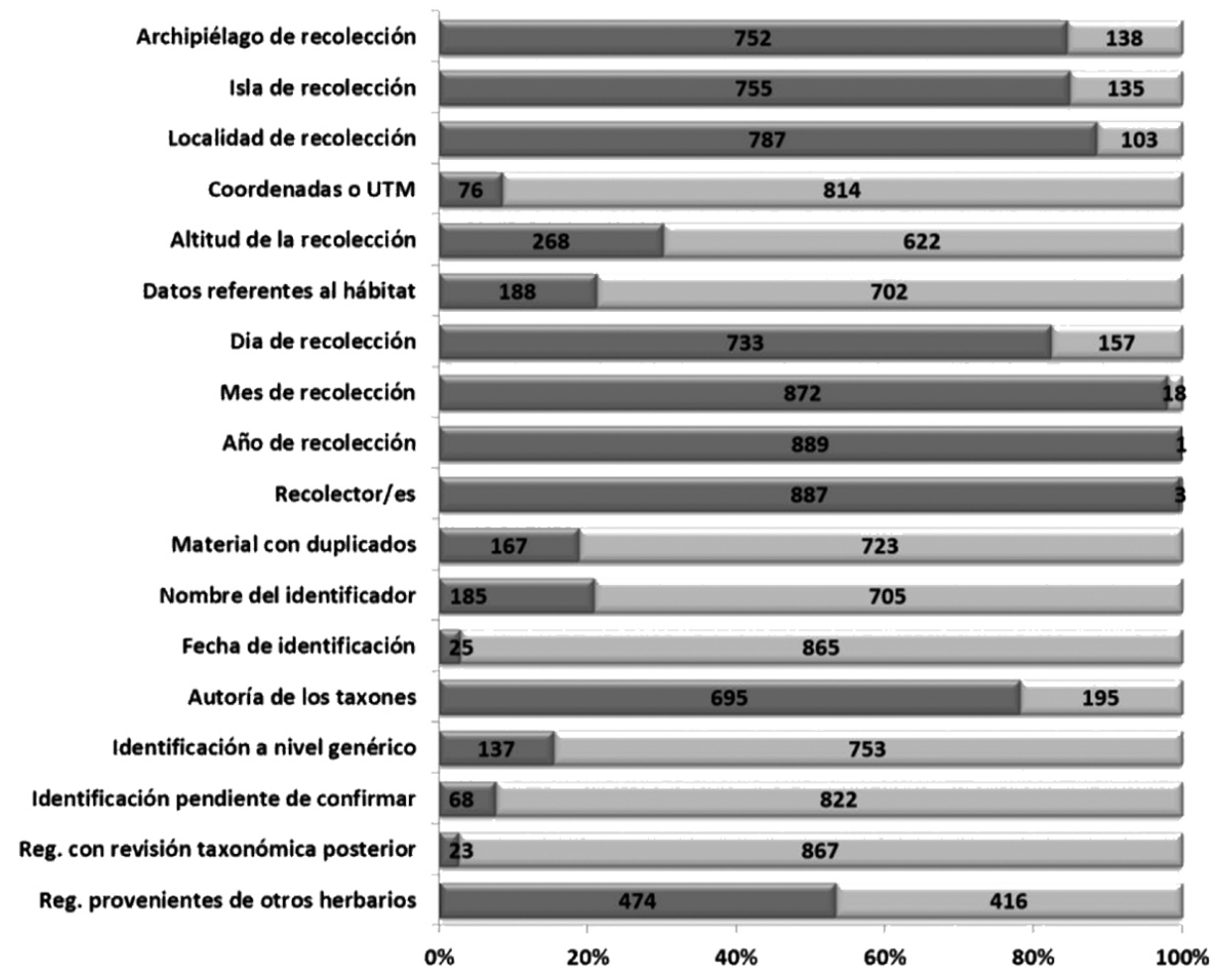

Figura 4. Calidad de los datos. Análisis de la calidad de los datos de recolección e identificación, número de duplicados recolectados, proveniencia del material de herbario y revisiones taxonómicas realizadas al material macaronésico presentes en el Herbario TFC. Se observan, por ejemplo, los elevados porcentajes presentes en las fechas de recolección y el nombre del recolector/es, mientras que destacan los bajos porcentajes de información referente al hábitat y a las coordenadas del lugar de recolección. Sería deseable que los primeros catorce marcadores (Archipiélago de recolección-Autoría de los taxones) presentaran porcentajes próximos al 100\%.

chmann et al. 1997). A estas recolecciones se les suman las de Cristóbal González-Coviella (1987) donadas al Herbario TFC (126 pliegos).

El volumen de especímenes de Madeira presentes en el Herbario TFC se debe, principalmente, a las recolecciones realizadas por el personal de Botánica de la Universidad de La Laguna, recogidas algunas de ellas en publicaciones de trabajos eminentemente taxonómicos (Del Arco 1982 y 1993, Del Arco et al., 1977, la Serna 1984, la Serna y Saenz 1981), y las de Ø.H. Rustan donadas por el Herbario O (Museo Botánico, Universidad de Oslo). Debemos reseńar la presencia del Holotypus de Micromeria varia Benth. subsp. thymoides (Solander ex Lowe) P. Pérez var. cacuminicolae P. Pérez, endemismo madeirense, recolectado en julio de 1976 por P. Pérez en "ex insula Maderae juxta Pico do Arrieiro» y cuyos duplicados (Isotypus) se encuentran depositados en su totalidad en herbarios institucionales fuera del ámbito 
macaronésico (Herbarios B, BM, FI, P y Z), tal y como reza en la etiqueta del espécimen. Actualmente este nombre ha sido considerado inválido por Puppo y Bräuchler (Puppo y Meimberg 2015) nominándolo Micromeria maderensis Puppo y Bräuchler. Por otro lado, una de las funciones que cumple el Herbario TFC es ser depositario del material testigo de trabajos de los grupos de investigación de la Universidad de La Laguna, sean producto de estudios taxonómicos o de otro tipo, caso del pliego de Artemisia gorgonum Webb (TFC 52 657) de Cabo Verde, estudiado en una tesis doctoral desarrollada en la ULL (Cosoveanu 2017), y del pliego de Lavandula pinnata L.f. (TFC 11 304; P-TFC 695) de Madeira, empleado en estudios palinológicos (León Arencibia y La Serna Ramos 1992).

Los máximos de productividad en cuanto al número de recolecciones realizadas corresponden a expediciones y al desarrollo de tesis doctorales con enfoque taxonómico, desarrolladas principalmente en la década de los 70: Acebes Ginovés y Pérez de Paz en Madeira y Salvajes (1976), Wildpret de la Torre, Pérez de Paz, Del Arco Aguilar y La Serna Ramos en Madeira (1977), Rustan en Madeira (1978) y Lobin en Cabo Verde (1978-1979). Posteriormente, en 1987, se dio un nuevo pico de recolección debido a la actividad desarrollada por González-Coviella en Cabo Verde. Los meses más exitosos en recolecciones corresponden fundamentalmente con la época favorable para el estudio botánico, final de primavera-inicio del verano, momento en el que las plantas presentan en estado óptimo los caracteres diagnósticos que permiten su identificación. El material recolectado en «febrero-marzo» corresponde, en su totalidad, a las recolecciones desarrolladas en el año 1976, fundamentalmente por los investigadores del Departamento de Botánica, Ecología y Fisiología Vegetal de la ULL Pedro Luis Pérez de Paz y Juan Ramón Acebes Ginovés, en el marco de la expedición «Agamenón 76» del 23 de febrero al 3 de marzo de 1976 al archipiélago de Salvajes (Acebes Ginovés y Pérez de Paz 1984 y Pérez de Paz y Acebes Ginovés 1983). En las últimas décadas las incorporaciones de material macaronésico en el Herbario TFC han disminuido considerablemente, fundamentalmente por la prácticamente ausencia de proyectos y tesis doctorales taxonómicas desarrolladas en el Área de Botánica, así como por la imposibilidad de realizar intercambios con otros herbarios de la región macaronésica debido a la falta de espacio para albergar nuevas recolecciones.

Aunque la colección estudiada sea pequeña en cuanto a número de especímenes, estos pueden ser en el futuro estudiados nuevamente y re-identificados, e incluso ser el material que ponga sobre la pista a los taxónomos sobre la presencia de nuevos taxones que habitan en estos archipiélagos, ya que sabemos que muchos nuevos taxones pasan desapercibidos en los herbarios incluso varias décadas antes de ser descubiertas y descritas como novedades científicas (Bebber et al. 2010). 


\section{AGRADECIMIENTOS}

A todos aquellos recolectores y herbarios institucionales que han permitido tener la actual colección de plantas vasculares de los archipiélagos de Azores, Madeira, Salvajes y Cabo Verde en el Herbario TFC. Agradecemos las aportaciones de dos revisores anónimos que han ayudado a mejorar el manuscrito.

\section{CONTRIBUCIÓN DE LOS AUTORES}

Conceptualización: CGM.

Metodología: CGM, MCLA y JRAG.

Análisis de datos: CGM.

Preparación del escrito original: CGM.

Corrección y edición del escrito definitivo: CGM, JRAG, MCLA y JARB.

Recibido: junio de 2019; ACEPTADo: febrero de 2020 


\section{REFERENCIAS}

Acebes Ginovés, J.R. y Pérez de Paz, P.L. 1984. Contribución al estudio de la flora y vegetación de las Islas Salvajes. Validaciones. Vieraea 14: 153-156.

AHIM. 2012. Normativa especifica sobre muestreos destructivos. Aprobada en la sesión ordinaria de la Asamblea General celebrada en Madrid, a 23 de noviembre de 2012 (https://ahim.wordpress.com/2013/11/07/normativa-especifica-sobre-muestreos-destructivos/ último acceso 1 de julio de 2019).

Baena, L. 2005. Tratamiento de las bases de datos del Herbario de la Universidad de Granada (GDA) como fuente de estudios de biodiversidad. Boletín de la AHIM 7: 12-15.

Bebber, D.P., Carine, M.A., Wood, J.R.I., Wortley, A.H., Harris, D.J., Prance, G.T., Davidse, G., Paige, J., Pennington, T.D., Robson, N.K.B. y Scotland, R.W. 2010. Herbaria are a major frontier for species discovery. PNAS 107: 22169-22171.

Brochmann, C., Rustan, Ø.H., Lobin, W. y Kilian, N. 1997. The endemic vascular plants of the Cape Verde Islands, W Africa. Sommerfeltia 24: 1-356.

Coello, J. 2007. Telesforo Bravo Expósito. Biografía de Cientificos Canarios, nº 7. Madrid: Dykinson 199 pp. Cosoveanu, A.D. 2017. Artemisia, an ark of bioactive endophytic fungi [Tesis doctoral]. Universidad de La Laguna. 284 pp.

Davis, C.C., Willis, C.G., Connolly, B., Kelly, C. y Ellison, A.M. 2015. Herbarium records are reliable sources of phenological change driven by climate and provide novel insights into species' phenological cueing mechanisms. Am. J. Bot. 102: 1599-1609.

Del Arco Aguilar, M.J. 1981. Revisión del género Teline Medicus (Fabaceae-Genisteae) en la Región Macaronésica. Estudio biométrico, taxonómico, iconográfico, corológico, ecológico y fitosociológico. [Tesis doctoral]. Universidad de La Laguna. 218 pp.

Del Arco Aguilar, M.J. 1982. Catálogo actualizado de los taxones del género Teline Medicus en la región macaronesica. Adiciones y nuevas combinaciones. Vieraea 12: 193-232.

Del Arco Aguilar, M.J. 1993. New combinations in the genus Teline Medicus. Typification of Cytisus pallidus Poiret. Itinera Geobot. 7: 519-523.

Del Arco Aguilar, M.J., Acebes Ginovés, J.R. y Wildpret de La Torre, W. 1977. Teline osyrioides (Svent.) Gibbs et Dingw. ssp. sericea (O. Kuntze) comb. et stat. nov., una nueva subespecie de la isla de Tenerife. Vieraea 7: 207-230.

Gallinat, A.S., Russo, L., Melaas, E.K., Willis, C.G. y Primack, R.B. 2018. Herbarium specimens show patterns of fruiting phenology in native and invasive plant species across New England. Am. J. Bot., 105: 31-41.

García-SÁnchez, J. y Cabezudo, B. 2013. Herbarium of the University of Malaga (Spain): Vascular Plants Collection. PhytoKeys 26: 7-19.

Grass, A., Tremetsberger, K., Hössinger, R. y Bernhardt, K.-G. 2014. Change of species and habitat diversity in the Pannonian Region of Eastern Lower Austria over 170 years: using herbarium records as a witness. Nat. Resour. J. 5: 583-596.

Heberling, J.M. e IsaAc, B.L. 2017. Herbarium specimens as exaptations: New uses for old collections. Am. J. Bot. 104: 963-965.

Holmes, M.W., Hammond, T.T., Wogan, G.O.U., Walsh, R.E., Labarbera, K., Wommack, E.A., Martins, F.M., Crawford, J.C., Mack, K.L., Bloch, L.M. y Nachman M.W. 2016. 
Natural history collections as windows on evolutionary processes. Mol. Ecol. 25: 864-881. DOI: $10.1111 / \mathrm{mec} .13529$.

Jardim, R. y Menezes de Sequeira, M. 2008. As plantas vasculares (Pteridophyta e Spermatophyta) dos Arquipélagos da Madaira e das Selvagens. En: Borges, P.A.V. et al., editores. Listagem dos fungos, flora e fauna terrestres dos arquipélagos da Madeira e Selvagens. Funchal and Angra do Heroísmo: Direcção Regional do Ambiente da Madeira and Universidade dos Açores, pp. 157-207.

La Serna, I. 1984. Revisión del género Bystropogon L'Hér. nom. cons. (Lamiaceae-Stachyoideae): Endemismo de la Región Macaronésica. Phanerogamarum Monographiae. Chicago: J. Cramer 18. $380 \mathrm{pp}$.

La Serna, I. y Saenz, C. 1981. Estudio morfológico del polen en el género Bystropogon L'Hér. (Lamiaceae). Bot. Macaronés. 8-9: 5-15.

Lavoie, C. 2013. Biological collections in an ever changing world: Herbaria as tools for biogeographical and environmental studies. Perspect. Plant Ecol. Evol. Syst. 15: 68-76.

León, M.C. 1982. Revisión del género Lavandula L. (Sec. Pterostachys Ging.) en la Región Macaronésica. [Tesis doctoral]. Universidad de La Laguna. 186 pp.

León, M.C. y La Serna, I. 1992. Palynological study of Lavandula (sect. Pterostoechas, Labiatae). Canario-madeirense endemics. Grana 31: 187-195.

LoвIN, W. 1982. Untersuchungen über Flora, Vegetation und biogeographische Beziehungen der Kapverdischen Inseln. Courier Forschungsinstitut Senckenberg, Band 53. [Tesis doctoral]. Universität Ulm. 112 pp.

Lobin, W. 1982. Beitrag zur Kenntnis der Cyperaceae (Phanerogamae, Monocotyledonae) der Kapverdischen Inseln. Cour. Forsch.-Inst. Senckenberg 53: 265-276.

Pérez de Paz, P.L. 1978. Revisión del género Micromeria Bentham (Lamiaceae-Stachyoideae) en la Región Macaronésica. Tenerife: Aula de cultura de Tenerife. Excmo. Cabildo Insular de Tenerife 306 pp.

Pérez de Paz, P.L. y Acebes Ginovés, J.R. 1983. Contribución al estudio de la flora y vegetación de las Islas Salvajes. Enn: Malato-Beliz, J. (editor) Comunicaçôes apresentadas ao II Congresso Internacional Pró Flora Macaronésica; Funchal pp. 221-268.

Puppo, P. y Meimberg, H. 2015. New species and new combinations in Micromeria (Lamiaceae) from the Canary Islands and Madeira. Phytotaxa 230: 001-021.

Rabeler, R.K., Svoboda, H.T., Thiers, B., Prather, L.A., Macklin, J.A., Lagomarsino, L.P., Majure, L.C y Ferguson, C.J. 2019. Herbarium Practices and Ethics, III. Syst. Bot. 44: 7-13.

Rich, T.C.G. 1998. Criteria for Evaluating the Importance of Herbarium Collections. The Biology Curator 13: 2-4.

Sánchez-Pinto, L., Rodríguez, M.L., Rodríguez, S, Martín, K., Cabrera, A. y Marrero, M.C. 2005. Pteridophyta, Spermatophyta. En: Arechavaleta, M. et al. (editores). Lista preliminar de especies silvestres de Cabo Verde (hongos, plantas y animales terrestres). 2005. Consejería de Medio Ambiente y Ordenación Territorial, Gobierno de Canarias pp. 38-57.

Shepherd, L.D. 2017. A non-destructive DNA sampling technique for herbarium specimens. PLoS ONE 12: e0183555.

Silva, L., Pinto, N., Press, B., Rumsey, F., Carine, M., Henderson, S. y Sjögren, E. 2005. Lista das Plantas Vaculares (Tracheobionta). En: Borges, P.A.V. et al. (editores) Listagem 
da Fauna (Mollusca e Artropoda) e Flora (Bryophyta, Pteridophyta e Spermatophyta). Terrestres dos Açores. Angra do Heroísmo and Ponta Delgada: Direcçao Regional do Ambiente e Universidade dos Açores pp. 117-146.

Smith, V.S. y Blagoderov, V. 2012. Bringing collections out of the dark. Zookeys 209: 1-6. Thiers, B. [actualizado continuamente]. Index Herbariorum: A global directory of public herbaria and associated staff. New York Botanical Garden's Virtual Herbarium. Disponible en: http:// sweetgum.nybg.org/science/ih/ (último acceso 30 de marzo de 2019).

Trujillo-Trujillo, E., Vargas-Triviño, P.A. y Salazar-Fajardo, L.V. 2014. Clasificación, manejo y conservación de colecciones biológicas: una mirada a su importancia para la biodiversidad. Mom. Cien. 11: 97-106.

Yoshida, K., Sasaki, E. y Kamoun, S. 2015. Computational analysis of ancient pathogen DNA from herbarium samples: challenges and prospects. Front. Plant Sci. 6: 771. 


\section{ANEXO I: LISTADO DE ESPECIES MACARONÉSICAS CON REPRESENTACIÓN EN EL HERBARIO TFC}

Taxones representados en el Herbario TFC de las islas de los archipiélagos de Azores (G: Graciosa, P: Pico, SJ: São Jorge, T: Terceira, SM: São Miguel), Madeira (M: Madeira, D: Desertas, PS: Porto Santo), Salvajes (SG: Salvaje Grande, GP: Gran Pitón) y Cabo Verde (SA: Santo Antão, SV: São Vicente, SN: São Nicolau, S: Sal, B: Boavista; M: Maio, T: Santiago, F: Fogo, Br: Brava). Se ha uniformado las paternidades de los taxones haciendo uso de la base de datos «International Plant Names Index (IPNI 2019)».

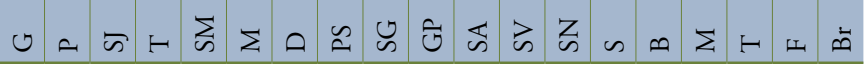

Abrus precatorius L.

Acanthospermum hispidum

DC.

Adenocarpus complicatus (L.)

J.Gay ex Gren. \& Godr.

Adiantum capillus-veneris $\mathrm{L}$.

Adiantum hispidulum Sw.

Ageratum conyzoides L.

Agropyron junceiforme

(Â.Löve et D.Löve) Á. Löve

et D.Löve

Aichryson divaricatum

(Aiton) Praeger

Aichryson villosum (Aiton)

Webb et Berthel.

Aizoon canariense L.

Ajuga iva (L.) Schreb. var. pseudoiva (Robill. et Cast. ex DC.) Robill. et Cast.

ex Bentham

Alternanthera caracasana

Humb., Bonpl. et Kunth

Alternanthera sessilis (L.)

DC.

Alysicarpus ovalifolius (Schu-

mach.) J.Léonard

Amaranthus hybridus L.

Anagallis arvensis L.

f. azurea Hyl.

Andrachne telephioides L. var. rotundifolia (C.A. Mey)

Muell. Arg. 


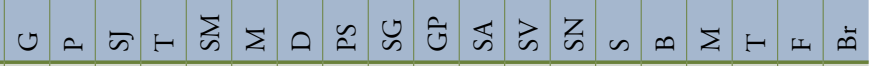

Andryala glandulosa Lam.

subsp. glandulosa

Anthyllis lemanniana Lowe

Arabis alpina L. subsp. cauca-

sica (Willd.) Briq.

Arachis hypogaea L.

Aristida adscensionis L.

Aristida funiculata Trin.

et Rupr.

Armeria maderensis Lowe

Artemisia argentea L'Hér.

Artemisia gorgonum

Webb in Hook.

Arthrocnemum glaucum

(Delarb.) Ung.-Stemb.

Asparagus nesiotes Svent.

Asparagus squarrosus

J.A. Schmidt

Asphodelus fistulosus L.

Asplenium billotii F. Schultz

Asplenium hemionitis L.

Asplenium marinum L.

Asplenium onopteris L. var.

onopteris

Asteriscus smithii (Webb)

Walp.

Asteriscus vogelii (Webb)

Walp.

Asterolinum linum-stellatum

(L.) Duby

Astragalus vogelii (Webb)

Bornm.

Astydamia latifolia (L.f.)

Baill.

Atriplex halimus L.

Bassia tomentosa (Lowe)

Maire et Weiller

Berberis maderensis Lowe

Beta procumbens C. Sm.

ex Hornem.

Bidens bipinnata L. 


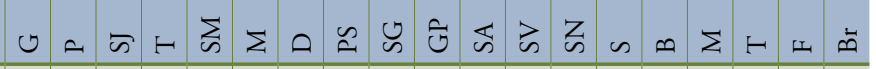

Bidens pilosa $\mathrm{L}$.

Blechnum spicant (L.) Roth

Boerhavia diffusa $\mathrm{L}$.

Borreria verticillata (L.)

G. Mey.

Brachypodium sylvaticum

(Huds.) P. Beauv.

Brassica nigra (L.)

W.D.J. Koch in Roehl

Briza maxima $\mathrm{L}$.

Bulbostylis barbata (Rottb.)

C.B. Clarke

Bystropogon canariensis (L.)

L'Hèr.

Bystropogon maderensis

Webb et Berthel.

Calamintha chenopodium

Roth.

Calamintha sylvatica Bromf.

subsp. ascendens (Jord.)

P.W. Ball

Calceolaria chelidonioides

Kunth

Calceolaria tripartita

Ruiz et Pav.

Calendula maderensis DC.

Calluna vulgaris (L.) Hull

Campanula jacobaea C. Sm.

ex Webb in Hook.

Campylanthus glaber Benth.

in DC.

Campylanthus spathulatus

A. Chev.

Carex antoniensis A. Chev.

Carlina salicifolia (L.f.) Cav.

Cassia absus L.

Cassia mimosoides L.

Cassia siamea Lam.

Cedronella canariensis (L.)

Webb et Berthel.

Cenchrus ciliaris $\mathrm{L}$.

Centaurea melitensis L.

\begin{tabular}{l|l|l|l|l|l}
\hline & & & & \\
\end{tabular}




$$
\text { U๘ங⿱ }
$$

Centaurium tenuiflorum

(Hoffmanns. et Link) Fritsch subsp. viridense (Bolle)

A. Hansen et Sunding

Cerastium glomeratum Thuill.

Chamaecytisus proliferus

(L.f.) Link

Chamaemeles coriacea Lindl.

Chelidonium majus L.

Chenopodium ambrosioides L.

Chenopodium murale L.

Christella dentata (Forssk.)

Brownsey et Jermy

Chrysanthemum pinnatifidum

L.f. var. thalassophilum Svent.

Cistanche phelypaea (L.)

Cout.

Cleome scaposa DC.

Cleome viscosa $\mathrm{L}$.

Clethra arborea Aiton

Clinopodium vulgare $\mathrm{L}$.

Clinopodium vulgare L. subsp. arundanum (Boiss.)

Nyman

Cocculus pendulus

(G. Forst.) Diels

Commelina benghalensis L.

Convolvulus massonii F. Dietr.

$+$

$+$

Conyza bonariensis (L.)

Cronquist

Conyza feae (Beg.) Wild

Conyza varia (Webb) Wild

Corchorus depressus (L.)

Stocks

Corchorus tridens L.

Crambe fruticosa L.f.

Cressa cretica L.

Crotalaria goreensis Guill.

et Perr. var. macrostipula

(Steud.) Bak. Fil.

Crotalaria microphylla Vahl 


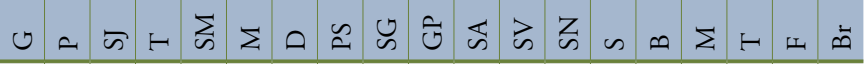

Crotalaria retusa L.

Crotalaria senegalensis Bacle

Cryptomeria japonica D. Don

Cuscuta epithymum (L.) L.

Cyperus alternifolius $\mathrm{L}$.

Cyperus amabilis Vahl

Cyperus articulatus $\mathrm{L}$.

Cyperus bulbosus Vahl

Cyperus cadamosti Bolle

Cyperus esculentus L.

Cyperus laevigatus $\mathrm{L}$.

Cyperus maritimus Poir.

Cyperus rotundus L.

Cystopteris fragilis (L.) Bernh.

Dactylis smithii Link

Dactylorhiza foliosa (Sol. ex Lowe) Soó

Dalechampia scandens L.

Desmodium scorpiurus

(Sw.) Desv.

Desmodium tortuosum

(Sw.) DC.

Dichanthium annulatum

(Forssk.) Stapf

Dicliptera verticillata

(Forssk.) C. Chr.

Digitalis purpurea L.

Diplotaxis glauca

(J.A. Schmidt) O.E. Schulz

Diplotaxis gracilis

(Webb) O.E. Schulz

Diplotaxis vogelii

(Webb) Cout.

Dolichos daltonii Webb

Echium candicans L.f.

Echium hypertropicum Webb

Echium stenosiphon Webb

Echium stenosiphon Webb subsp. stenosiphon

\section{$+$}

$++\quad+$

$+$

$+\quad+$

$+$ 


$$
\text { Uニ }
$$

Eclipta prostrata (L.) L.

Egeria densa Planch.

Elaphoglossum pahaceum

(Hooker et Grev.) Sledge

Elvira biflora (L.) L.

Enneapogon desvauxii $\mathrm{Sm}$.

Epilobium hirsutum L.

Equisetum ramosissimum

Desf.

Eremopogon foveolatus

(Delile) Stapf

Erica arborea L.

Erica maderensis

(Benth.) Bornm.

Erysimum bicolor

(Hornem.) DC.

Erysimum caboverdeanum

(A.Chev.) Sunding

Euphorbia forskaolii J. Gay

Euphorbia hirta L.

Euphorbia prostrata Aiton

Evolvulus alsinoides L.

Fagonia cretica L.

Fagonia isotricha Murb.

Festuca albida Lowe

Fimbristylis debilis Steud.

Fimbristylis ferruginea

(L.) Vahl

$+$

Fimbristylis hispidula

(Vahl) Kunth

Flaveria bidentis (L.) Kuntze

Forsskaolea procridifolia Webb

Frankenia ericifolia C. Sm.

Frankenia ericifolia

C. Sm. var. ericifolia

Frankenia laevis L.

Frankenia pulverulenta L.

Fuirena ciliaris (L.) Roxb.

Galinsoga ciliata (Rafin)

S.F. Blake

$++$

$+$

$+\quad+$

\begin{tabular}{|l|l|l|l|l|l|l|}
\hline & & & & + & + \\
\hline & & & & & & \\
\hline
\end{tabular}

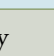

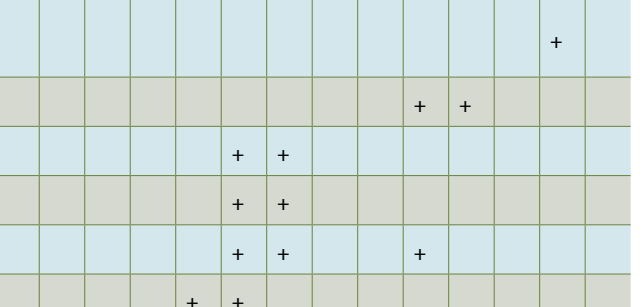




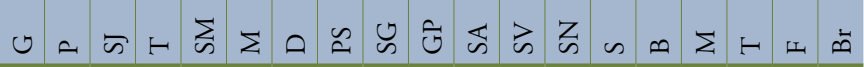

Galinsoga parviftora Cav.

Galium parisiense L.

Galium productum Lowe

Galium scabrum L.

Genista tenera (Jacq. ex

Murray) Kuntze

Gennaria diphylla

(Link) Parl.

Geranium palmatum Cav.

Glinus lotoides L.

Globularia amygdalifolia

Webb

Globularia salicina Lam.

Gnaphalium luteo-album L.

Gnidia polystachya

P.J. Bergius

Gynandropsis gynandra

(L.) Briq.

Helianthemum gorgoneum

Webb

Helichrysum melaleucum

Rchb. ex Holl

Heliotropium curassavicum L.

Heliotropium ramosissimum

(Lehm.) DC.

Heteropogon contortus (L.)

Roem. et Schult.

Huperzia selago (L.) Bernh. ex Schrank et Mart. subsp. dentata (Herter) Valentine

Hymenophyllum tunbrigense (L.) $\mathrm{Sm}$.

Hyparrhenia hirta (L.) Stapf

Hypericum elodes L.

Hypericum foliosum Aiton

Hypodematium crenatum

(Forssk.) Kuhn

Indigofera colutea (Burm. f.)

Merr.

Indigofera cordifolia

B. Heyne ex Roth

Indigofera hirsuta L. 
Indigofera parviflora

B. Heyne ex Wight et Arn.

Indigofera senegalensis Lam.

Indigofera subulata Poir.

Ipomoea cairica (L.) Sweet

Ipomoea coptica (L.) Roth

ex Roem. et Schult.

Ipomoea eriocarpa $\mathrm{R}$. Br.

Jasminum odoratissimum $\mathrm{L}$.

Juncus acutus L.

Kickxia brunneri (Benth.)

Janch.

Kickxia dichondrifolia

(Benth.) Sunding

Kohautia aspera (Heyne ex Roth) Bremek.

Kyllinga pumila Michx.

Kyllinga squamulata Thonn. ex Vahl

Launaea arborescens (Batt.)

Murb.

Launaea nudicaulis (L.)

Hook. f.

Launaea picridioides

(Webb) Engl.

Laurus azorica (Seub.) Franco

Lavandula dentata $\mathrm{L}$.

Lavandula dentata L. var. rendalliana Bolle

Lavandula latifolia Medik.

Lavandula pinnata L.f.

Lavandula rotundifolia

Benth.

Lavandula rotundifolia

Benth. var. subpinnatifida

Chev.

Lavandula stricta Delarb.

Leontodon rigens (Dryand.)

Paiva et Ormonde

Lepidium sativum L.

Limonium braunii (Bolle)

A. Chev. 


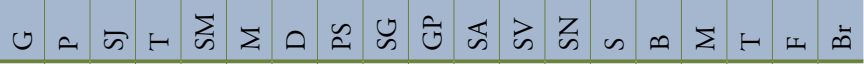

Limonium jovibarba (Webb)

Kuntze

Limonium papillatum (Webb et Berth.) O. Kuntze var.

callibotryum Svent.

Limonium vulgare Mill. subsp. serotinum (Rchb.)

Gems.

Linum trigynum $\mathrm{L}$.

Lobelia urens L.

Lobularia canariensis (DC.)

L. Borgen subsp. succulenta

L. Borgen

Lobularia canariensis (DC.)

L. Borgen aff. subsp. succulenta L. Borgen

Lobularia canariensis (DC.)

L. Borgen subsp. fruticosa

(Webb) L. Borgen

Lophochloa cristata (L.)

H. Hyl.

Lotononis platycarpa (Viv.)

Pic.Serm.

Lotus arborescens Lowe ex

Cout.

Lotus brunneri Webb in

Hook.

Lotus glaucus Aiton

Lotus glaucus Aiton var.

glaucus

Lotus jacobaeus L.

Lotus loweanus Webb et

Berthel.

Lotus salvagensis R.P. Murray

Lotus uliginosus Schkuhr

Luzula multiflora (Retz.) Lej.

Lycopersicon esculentum Mill.

Lycopodiella cernua (L.) Pic.

Serm.

Lysimachia azorica Hornem. ex Hook.

Mariscus alternifolius Vahl

Mariscus squarrosus (L.)

C.B. Clarke

$+$




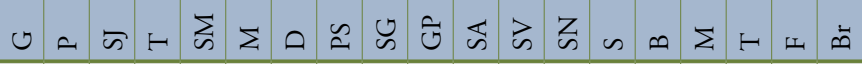

Maytenus dryandri (Lowe)

Loes.

Melanoselinum decipiens

(Schrad. et J.C. Wendl.)

Hoffm.

Melhania ovata (Cav.)

Spreng.

Mesembryanthemum

nodiflorum L.

Micromeria forbesii Benth.

Micromeria forbesii Benth. var. altitudinum Bolle

Micromeria forbesii Benth. var. inodora Schmidt

Micromeria varia Benth. subsp. thymoides (Sol. ex Lowe) P. Pérez

Micromeria varia Benth. subsp. thymoides (Sol. ex Lowe) P. Pérez var. cacuminicolae P. Pérez

Micromeria varia Benth. subsp. thymoides (Sol. ex Lowe) P. Pérez var. thymoides

Misopates orontium (L.) Raf.

Mitracarpus villosus (Sw.)

DC.

Mollugo nudicaulis Lam.

Mollugo verticillata $\mathrm{L}$.

Musschia aurea (L.f.)

Dumort.

Myosotis discolor Pers. subsp. canariensis (Pit.) Grau

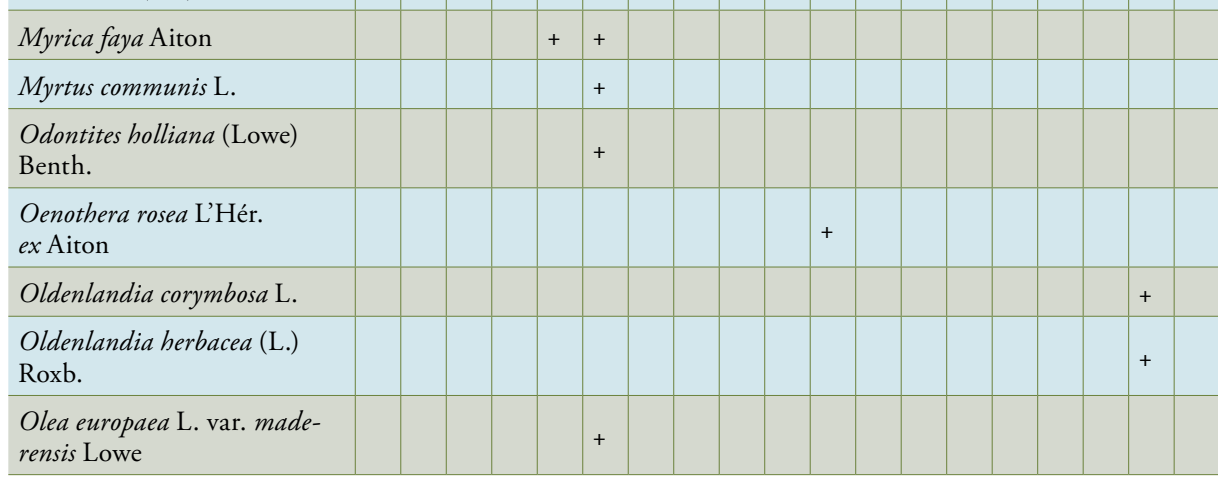




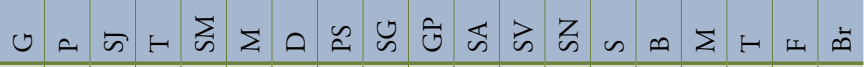

Origanum virens Hoffmanns.

et Link

Parietaria debilis G.Forst.

Paronychia illecebroides

(C. Sm. ex Webb) Webb

Paronychia illecebroides

(C.Sm. ex Webb) Webb

var. illecebroides

Patellifolia procumbens

(C. Sm. ex Hornem.)

A.J.Scott, Ford-Lloyd et

J.T. Williams

Pegolettia senegalensis Cass.

Pericallis aurita (L'Hér.)

B. Nord.

\section{Periploca chevalieri Browicz \\ Peristrophe bicalyculata \\ (Retz.) Nees}

Phagnalon melanoleucum

Webb in Hook.

Phyllanthus amarus Schumach. et Thonn.

\section{Phyllanthus rotundifolius}

J.G. Klein ex Willd.

Phyllis nobla L.

Phytolacca americana L.

Pittosporum undulatum Vent.

Plantago arborescens Poir.

Plantago arborescens Poir. subsp. maderensis (Decne.)

A. Hansen et G. Kunkel

Plantago coronopus L.

Plantago psyllium L. var.

obtusata Svent.

Plantago subspathulata Pilg.

Pluchea ovalis (Pers.) DC.

Plumbago zeylanica L.

Polycarpaea gayi Webb

$+$

$+$

$+$

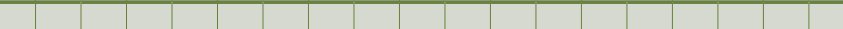

\begin{tabular}{ll|l|l|l} 
& & & & \\
\end{tabular}




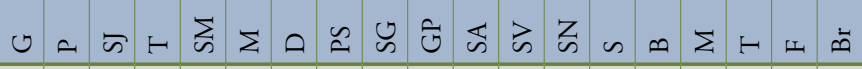

Polygonum capitatum Buch.-

Ham. ex D. Don

Polygonum maritimum L.

Polygonum salicifolium

Brouss. ex Willd.

Polypogon maritimus Willd.

Polypogon monspeliensis (L.)

Desf.

Polypogon semiverticillatus

(Forssk.) Hyl.

Polypogon viridis (Gouan)

Breistr.

Potamogeton antaicus Hagstr.

Prasium majus L.

Prunella vulgaris L.

Pulicaria burchardii Hutch.

subsp. longifolia

E. Gamal-Eldin

Pycreus mundtii Nees

Pycreus polystachyos (Rottb.)

P. Beauv.

Rhamnus glandulosa Aiton

Rhynchosia minima (L.) DC.

Rhynchosia minima (L.)

DC. var. memnonia (Delile)

T. Cooke

Rubia fruticosa Aiton

Rumex maderensis Lowe

Ruppia maritima L.

Ruscus streptophyllus Yeo

Salsola kali L.

Salvia aegyptiaca L.

Salvia coccinea Juss. ex

Murray

Sambucus ebulus L.

Samolus valerandi $\mathrm{L}$.

Sarcostemma daltonii Decne.

Saxifraga maderensis D. Don

Schizogyne sericea (L.f.) DC.

Schoenefeldia gracilis Kunth 


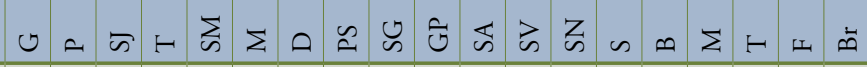

Scirpus grandispicus (Steud.)

Berhaut

Sclerocephalus arabicus Boiss.

Scrophularia arguta Soland.

Scrophularia scorodonia L.

Sedum farinosum Lowe

Sedum nudum Aiton subsp. nudum

Semele androgyna (L.) Kunth

Senecio incrassatus Lowe

Senecio maderensis DC.

Sesbania bispinosa (Jacq.)

W. Wight

Setaria adhaerens (Forssk.)

Chiov.

Setaria pumila (Poir.) Roem. et Schult.

Sibthorpia peregrina L.

Sideritis candicans Aiton

Sideritis candicans Aiton var.

candicans

Sideroxylon marmulano

Banks ex Lowe

Sideroxylon marmulano

Banks ex Lowe var. marmu-

lano

Silene gallica L.

Sinapidendron angustifolium

(DC.) Lowe

Sinapidendron frutescens (Sol.

in Aiton) Lowe var. frutescens

Sinapidendron rupestre Lowe

Sonchus fruticosus L.f.

Sonchus oleraceus L.

$+$

$+$

$+$

$+$

Sorbus maderensis (Lowe)

Dode

Spergularia azorica (Kindb.)

Lebel

Spergularia fallax Lowe

Borreria verticillata (L.)

G. Mey.

$+$

$+$

\begin{tabular}{l|l|l|l|l|l|l|l|l|l|l}
\hline & & & & + & & & & & & \\
\hline
\end{tabular}

$+$

$+$

$+$

$++$

$+$ 


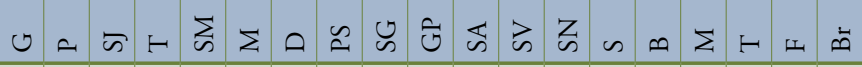

Sporobolus minutus Link

Sporobolus spicatus (Vahl)

Kunth

Stylosanthes fruticosa (Retz.)

Alston

Suaeda vera Forssk.

ex J.F. Gmel

Synedrella nodiflora (L.)

Gaertn.

Tagetes minuta L.

Tamarix canariensis Willd.

Tamarix gallica L.

Tamarix senegalensis DC.

Teline maderensis Webb et Berthel.

Teline maderensis Webb et

Berthel. var. maderensis

Teline maderensis Webb et

Berthel. var. paivae (Lowe)

del Arco

Tephrosia bracteolata Guill. et Perr.

Tephrosia pedicellata Baker in Oliv.

Tephrosia purpurea (L.) Pers. subsp. leptostachya (DC.)

Brummitt

Tephrosia uniflora Pers.

Teucrium abutiloides L'Hér.

Teucrium betonicum L'Hér.

Thymus caespititius Brot.

Tolpis azorica (Nutt.) P. Silva

Tolpis fruticosa Schrank

Tolpis succulenta (Dryand.

in Aiton) Lowe

Tornabenea hirta J.A.Schmidt

Trachelium caeruleum $\mathrm{L}$.

Tribulus cistoides L.

Trichodesma africanum (L.)

Lehm.

Tricholaena teneriffae (L.f.)

Link 


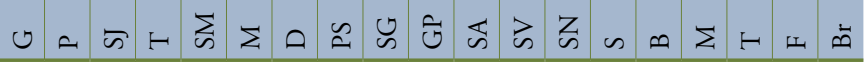

Trichomanes speciosum Willd.

Trifolium glomeratum $\mathrm{L}$.

Triumfetta pentandra A.Rich.

Umbilicus rupestris (Salisb.)

Dandy

Urospermum picroides (L.)

Scop. ex F.W. Schmidt

Urtica membranacea Poir.

Vaccinium maderense Link

Vaccinium padifolium $\mathrm{Sm}$.

Vandenboschia speciosa

(Willd.) Copel

Verbascum capitis-viridis

Hub.-Mor.

Vicia capreolata Lowe

Vicia leptoclada (Webb)

Dandy

Vicia sativa L.

Vicia tenuissima (Bieb.)

Schinz. et Thell.

Viola paradoxa Lowe

Viola riviniana $\mathrm{Rchb}$

Visnea mocanera L.f.

Wahlenbergia lobelioides

(L.f.) Link

Wahlenbergia lobelioides (L.f.)

A. DC. subsp. lobelioides

Waltheria indica $\mathrm{L}$.

Zaleya pentandra (L.)

C. Jeffrey

Zinnia peruviana (L.) L.

Ziziphus mauritiana Lam.

Zornia glochidiata Rchb.

ex DC.

Zygophyllum fontanesii Webb et Berthel.

Zygophyllum simplex $\mathrm{L}$.

$+$

$+$

$+$

$+$

$+\quad+$

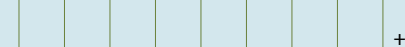

$+$

$+$

$+$

$+$

$+$ 